\title{
Alveolar Ridge Preservation in Mandibular Molars Using Mixture of Autogenous Bone and Anorganic Bovine Bone (ABB) Versus Anorganic Bovine Bone Alone Versus Absorbent Gelatin Sponge (Randomized Clinical Trial)
}

\author{
Dina Mohammed Alesawy ${ }^{1 *}$, Nevien Abd El-Latif Askar ${ }^{2}$, Mohamed \\ Atef Abdel-Rasoul ${ }^{2}$ and Hatem Abdel-Fattah Amer $^{3}$ \\ ${ }^{1}$ Department of Oral Implantology, Faculty of Dentistry - Cairo University, Egypt \\ ${ }^{2}$ Department of Oral and Maxillofacial Surgery, Faculty of Dentistry - Cairo University, \\ Egypt \\ ${ }^{3}$ Department of Oral and Maxillofacial Pathology, Faculty of Dentistry - Cairo \\ University, Egypt \\ *Corresponding Author: Dina Mohammed Alesawy, Department of Oral \\ Implantology, Faculty of Dentistry - Cairo University, Egypt.
}

Received: December 09, 2020

Published: December 29, 2020

(C) All rights are reserved by Dina

Mohammed Alesawy., et al.

\begin{abstract}
Purpose: Radiographic and Histomorphometric assessment of Autogenous and Anorganic bovine bone graft mixture (Auto/ABB) vs Anorganic Bovine Bone (ABB) vs Absorbable gelatin sponge in alveolar socket preservation.

Materials and Methods: 30 patients, 1/group required extraction of mandibular molar tooth. Immediate and 6 months post grafting CBCT radiographs. Crestal flaps, followed by core biopsy. Histomorphometric analysis of mean bone/area and residual percent and radiographical evaluation of bone loss.

Results: Auto/ABB loss buccally $2.64 \pm 1.48 \mathrm{~mm}$, lingually $1.59 \pm 0.86 \mathrm{~mm}$, width $1.63 \pm 0.97 \mathrm{~mm}$. Mean bone area percent $42.34 \%$ residual material percent $32.19 \%$. The ABB loss buccally $1.62 \mathrm{~mm}$, lingually $0.48 \mathrm{~mm}$ and width $1.55 \mathrm{~mm}$. Bone area percent was $48.42 \%$ and residual percent of $20.87 \%$. The AGS recorded a buccal loss of $1.29 \mathrm{~mm}$, lingual $1.44 \mathrm{~mm}$ and horizontal $0.89 \mathrm{~mm}$. The Bone area percent $58.88 \%$ and a residual of $20.36 \%$.

Conclusion: The Auto/ABB loss was highest in all the radiographical dimensions with the least bone area percent and the highest residual material present giving rise to questioning its effectiveness in socket preservation. ABB had the significant least amount of lingual loss of bone. The AGS showed promising results with the bone area percent recorded the most and the least residual material percent.
\end{abstract}

Keywords: Autogenous; Anorganic Bovine; Alveolar Bone

\section{Introduction}

Alveolar bone atrophy post-extraction has been well documented in the past years. Normal healing event results in a minimal loss of vertical height (around $1 \mathrm{~mm}$ ), but a substantial loss of width in the buccal-lingual plane (4-6 mm) [1]. Clinical measurements show a three-dimensional loss of alveolar ridge volume of approx. 35 per cent in the first three months and 50 per cent in the first six months. An additional negative side-effect of this resorption process is the simultaneous reduction and shift of the crestal keratinized gingiva [1].

With the expanding literature documentation about the immediate implant installation in fresh extraction sockets, the loss of 
alveolar bone from post-extraction remodeling were greatly decreased even though not completely inhibited [3]. The outfall of this modality is when there is a contraindication that would prevent the implant placement. Such contraindications could be patients related such as cost, duration, health factors of patient while others are extracted socket related factors that would prevent the immediate installation of the implant. One of the most common example that were found is that the socket is too large to accept an implant and allow for primary stability achievement due to infection or socket defects from extraction [2].

The large socket could be due to previous infection in socket that resulted in surrounding bone resorption, the extracted tooth roots were too large or fused that doesn't allow for interseptal bone implant installation, in addition to the anatomical landmarks in close relation to the extracted tooth that do not allow for apical extension of the implant osteotomy site to provide required primary stability [3]. The inability to surgically install a prosthetically driven implant into the extraction socket while still achieving optimum primary stability would obligate the postponing of the implant insertion for a duration of socket healing and demand a way to preserve the socket from collapsing and the later need for bone regeneration procedures [2].

According to the previous situations the most common tooth that was found to meet these contraindications was seen to be the posterior lower molars. Since the roots are mostly large, the interseptal bone dimension is often found to be thin, a variety of malformations of the ridges and the inferior alveolar nerve proximity does not allow for apical osteotomy preparation with little or no possibility of reinforcement via bi-cortical stabilization thus, the unavoidable waiting for implant placement until after partial or complete socket healing [4].

\section{Alveolar socket healing post extraction}

According to previous investigations it was reported the width of the alveolar ridge reduced up to $50 \%$ during the 12 month observation period after the extraction. This loss corresponded to a buccal-lingual change in dimension of 4.5 to $6.1 \mathrm{~mm}$. The finding that approximately two thirds of this reduction occurred within the first 3 months after tooth extraction also corresponded to earlier studies. The percentage reduction was larger at molar sites compared to premolar sites. Furthermore, an average of 0.5 to 0.9 $\mathrm{mm}$ of vertical bone resorption can be expected by 12 months [5].
The reduction of alveolar bone in edentulous sites was also found to be related to the cause of extraction, the trauma subjected during tooth removal such as amount of bone damage during extraction or mucoperiosteal flab elevation, and wither the edentulous site is temporarily restored post-extraction or not and the type of prosthetics involved in its restoration [6].

The anatomical factors were found to be of relevance in the alveolar bone resorption mode the facial form, mandible size, bone quality, blood supply and muscle attachment were all variables in the attitude of bone remodelling [7]. The difference in bone resistance to resorption tolerance, and stability has been historically discussed in the literature [8] metabolic, functional, and prosthetic factors. Anatomic factors include the size, shape, and density of ridges, the thickness and character of the mucosal covering, the ridge relationships, and the number and depth of sockets. Metabolic factors include all of the multiple nutritional, hormonal, and other metabolic factors which influence the relative cellular activity of the bone-forming cells (osteoblasts. According to the inflammatory reaction created before and during the extraction from the trauma inflicted within the residual ridge the metabolic process of the bone healing varies, this include inflammatory bone diseases and presence of local inflammatory mediators [9]. According the review, Systemically the age and gender of the patient in addition to their bone regulatory hormones and dietary calcium levels are of great effect to the speed of resorption [10].

\section{Alveolar socket preservation}

Regenerative techniques have been widely tested in controlled and uncontrolled studies with various materials and clinical approaches: bone grafting alone, including autografts, allografts $[11,12]$. Xenografts $[13,14]$ and alloplasts $[15,16]$; membrane alone, whether absorbable [17] or not [18,19]; and membrane in conjunction with grafting [20-22].

The three different categories of socket manipulation are graft alone, graft with barrier or barrier alone were all found to be better than spontaneous socket healing, but the statistical significance showed better results in the use of barriers alone than grafts alone or graft with barrier gave doubts that led to our research in this thesis.

The argue was that the barrier provided space for the blood clot inside the socket were protected and the space provided gave a 
chance for shielding the socket from outsider influences while still provided the socket healing with normal bone with no additives or bone quality difference than normal healing bone. Thus, the idea of using the absorbent gel foam in the socket healing to facilitate the blood clot stability inside the socket while sealing the socket access from external influences came and comparing it with bone grafting in relation to the quality of the produced bone histopathologically.

In evaluating the cost/benefit ratio the major cost expenses on bone grafting material were put into mind and the fact that some of the patients reasons to postpone the implant placement were financial reasons so the facility of the preservation procedure were put into consideration for both a general practitioner that needs to perform the preservation technique and cost effectiveness for the patient who require it. Thus, the most available, applicable and affordable materials were thought to be researched according to the preservation that could be achieved with each, the quality of bone that would be produced from the preservation procedure and the effectiveness of this bone quality for later implant placement and implant stability.

The biological mechanisms of graft actions are nominated below:

- Osteoconduction: Where the graft material only acts as a scaffold to keep the space for the native new bone formation. Osteoblasts from the margin of defect that is being grafted, utilize the bone graft material as a framework upon which to spread and generate new bone [23]. This is considered the least form of grafts. Clinically, osteoconduction results in bone growth within a defect or on a surface which may otherwise repair with soft tissue. Osteoconductive properties are related to structural and material properties (porosity, pore size, shape, particle size, crystallinity) that influence cell attachment, migration, differentiation and vascularization. Examples of purely osteoconductive materials include hydroxylapatite (natural or synthetic), polymers, and bioactive glass [24].

- Osteoinduction: Where the graft material is responsible to stimulate the host cells to differentiate into osteoblasts and thus format new bone. primitive, undifferentiated and pluripotent cells are somehow stimulated to develop into the bone-forming cell lineage [25]. Osteoinductivity is dependent on the activity of bone morphogenetic proteins (BMP's) which are located within the organic matrix of bone. Demineralized freeze-dried human bone allograft is an example of an osteoinductive material, although the osteoinductive capacity can be quite variable. Growth factors, such as recombinant human growth factor (rh-BMP2), which has only recently become available for clinical use, would also be considered osteoinductive.

- Osteopromotion: Where the graft material promote osteoinduction but lack the Osteoinductive mediators [26].

- Osteogenesis: Where the graft material contains vital osteoblastic cells that starts to form new bone in the recipient site on its own.

\section{Autogenous bone graft}

Autogenous bone is known as the golden graft. It has all the required properties for the bone graft to succeed, cost barely nothing, and with no adverse reactions to be expected, no antigenic properties and with zero risk of disease transmission. The only drawback of the autogenous bone is the need for a secondary surgical site to acquire the graft and the limited amount of graft material that could be acquired from intraoral sites. From a biological standpoint, the autogenous bone is the only ideal bone graft material [24]. Being Osteoconductive [27], Osteoinductive [25], Osteogenic.

Depending on the location of the donor site, it may be cortical or cancellous. Cancellous bone contains a higher percentage of cells, and therefore has more osteogenic potential. Conversely, while there are fewer cells, cortical bone is believed to have higher levels of bone morphogenic proteins (BMP's) and is useful when immediate structural support or three-dimensional augmentation is required [28]. Cancellous grafts are revascularized more rapidly and completely than cortical grafts. Gradual revascularization occurs at a rate of about $1 \mathrm{~mm}$ per day with cancellous grafts revascularizing in approximately 2 weeks, whereas cortical grafts may take 2 months or longer to revascularize [29].

Autogenous bone may be prepared and used as granules, shavings or blocks depending on availability and the clinical requirements. A major advantage of autogenous bone is the presence of 
viable osteogenic cells within the graft. However, only a small percentage of these cells actually survive transplantation. The remaining non-vital bone matrix serves as an osteoconductive scaffold and is gradually replaced by "creeping substitution" [30] which is a process of osteoclastic resorption followed by osteoblastic deposition of new bone. Gradual revascularization occurs at a rate of about $1 \mathrm{~mm}$ per day with cancellous grafts revascularizing in approximately 2 weeks, whereas cortical grafts may take 2 months or longer to revascularize [31]. As autogenous bone matrix is broken down during remodeling by osteoclasts, bone morphogenetic proteins are released resulting in the attraction, differentiation and proliferation of bone forming cells [32].

Autogenous graft and anorganic bovine bone graft mixture

The first documented attempts to mix xenogeneic bone graft with autogenous bone was in 1978 after the successful results of xenografts alone as a bone grafting material. The autogenous bone role plays in the induction of the osteogenesis process, cancellous graft is known to have the highest osteogenic potential due to the large number of osteoblasts and undifferentiated mesenchymal cells. The early grafting histological studies showed that a large part of these cells dies after transplantation and only superficial parts survive that gets the blood supply from the host the earliest, that means the inner cells osteogenesis turns to be an osteoinductive potential only as they attract the host cells to differentiate and starts the bone repair process, thus the idea of putting the osteoinductive $\mathrm{ABB}$ cells from the beginning to aid in osteoinduction while preserve the host from collapse and stabilize the autografts for osteogenesis.

The reason for mixing the autogenous bone with the $A B B$ is that the composite mixture provides the advantageous characteristics of both grafts, the second surgical site is not required to be a major traumatic surgery, the graft material remains to have the osteogenic properties while still the amount of resorption and host site collapse is greatly decreased by the slow resorbability of the ABB. It's also timesaving, easy, practical and safe with a high success rate and avoidance of large complications of major donor site surgeries. The mixture of the graft materials for socket grafting require a very small amount of autogenous graft that could be easily acquired intraorally from multiple sites like the tuberosity, chin, buccal shelf or even a close site in the ridge with a previously missing tooth that does not require rehabilitation [33].

\section{Absorbant gelatin sponge (AGS)}

Absorbant gelatin sponge are gelfoam dental sponges that was originally designed in 1945 by Upjohn Company, a pharmaceutical manufacturing firm [34]. In 1947, Council on Pharmacy and Chemistry announced that -Absorbable gelatin sponge material, although insoluble in aqueous mediums, is absorbable and as such may be used as a surgical sponge, which may be left in place following closure of an operative wound. It is claimed that such material will be completely absorbed without inducing excessive formation of scar tissue or excessive cellular reaction in from four to six weeks [35].

It is one of the more commonly employed agents for control of minor bleeding. Gelatin is a thermal-denatured collagen [36]. They are small, sterile, surgical sponges prepared from specially treated and purified gelatin solution or a sterilized porcine skin gelatin that may be prepared either by acidic (type A) or alkaline (type B) treatments (extraction) of bovine or porcine skin, bone or tendon, followed by heat-treatment in an aqueous environment (hydrolysis) [37]but were thought to be too costly for commercial production. Yeast have been engineered to express high levels of type I homotrimer and heterotrimer and type II and type III collagen. Coexpression of collagen genes and cDNAs encoding the subunits of prolyl hydroxylase has lead to the synthesis of completely hydroxylated, thermostable collagens. Human types I and III collagen homotrimers have been expressed in transgenic tobacco plants, while transgenic mice have been engineered to produce full-length type I procollagen homotrimer as well as a $\alpha 2$ (I, which is beaten to desired porosity, dried, sectioned, packaged, sealed, and sterilized by dry heat or gamma rays, The final product is separated from bouillon using various methods such as settling, filtering, and centrifuging [38]. Gelatin has a long history of usage in the food industry as a clarification agent, stabilizer and protective coating material, and in the pharmaceutical industry for manufacturing capsules, ointments, cosmetics, tablet coating, and emulsion [39]. Due to its hemostatic properties, gelatin sponge has been widely used in surgery as a wound dressing, adhesive and absorbent pad. 
The advantage of gelatin over collagen matrix is its ease of extraction and preparation, which results in a cheaper and high quantity production of gelatin matrix [40]. Furthermore, unlike collagen, gelatin does not express any antigenicity in physiological conditions [41]. Gelfoam is pliable and is capable of absorbing and holding within its meshes many times its weight in whole blood so its use as a hemostatic device [38]. When implanted in tissues, gelfoam dental sponges are completely absorbed within four to six weeks without inducing excessive scar tissue [42]. The mode of action of AGS is related to formation of a mechanical matrix that facilitates clotting rather than affecting the blood clotting mechanism [43]. The gelatin sponge material induces platelet adhesion and releases the content of the $\alpha$-granules which are important for the performance of hemostatic sponges in vivo. It is also proved neither cytotoxic nor genotoxic [44].

As the sponge absorbs blood it swells and fills the cavity where it was placed providing structural support to prevent the collapse of soft tissue [45]. The resorbable dressing helps in the formation of the matrix clot that would ensure natural organization of the healing cells to facilitate healing by primary intentions [46], accelerate the socket healing and aids in ridge dimensional maintenance, thus reducing postoperative complications and correlatively postoperative treatment needs, in addition to the reduction of primary and secondary hemorrhage due to the hemostatic effect of the gelatin sponge rapidly forming the blood clot on surgical site [42].

Rohanizadeh., et al. [47] investigated the ability of gelatin scaffolds to be used as a support for osteoblast proliferation and differentiation and reported that tested scaffolds demonstrated the ability to support osteoblast activities and allowed cell proliferation and cell migration into the sponge porosities. He found out that because of its flexibility, biocompatibility, and biodegradability, has the potential to be used as a scaffold to support osteoblasts and to promote bone regeneration in defective areas [47]. The residuals of gelatin sponge are proofed to be incorporated in the new bone without the presence of any significant foreign-body $[48,49] 18 \mathrm{fe}$ males.

\section{Aim of Study}

To assess the quality and quantity of regenerated bone using Autogenous and Anorganic bovine bone graft mixture vs the An- organic Bovine Bone alone vs the use of absorbable gelatin sponge alone for alveolar socket preservation.

\section{Materials and Methods}

A total of 36 patients (25 males, 16 females; average age 20 to 60 years) was enrolled in this study, the patients were randomly divided into 3 equal groups using block randomization with stratification (block size: 4) using a formula on Microsoft Excel Software. the mean follow-up was of 6 months from the extraction and socket preservation (1st stage) followed by implant placement, 6-month of follow-up then prosthetic stage and delivery ( $2^{\text {nd }}$ stage) and 1 year follow up for the implants and prosthetics ( $3^{\text {rd }}$ stage).

2 patients from each group were dropped out of the experiment through the first stage as they preferred to postpone the implant placement for different personal reasons which would have affected the 6-month result analysis for the experiment. Making a total of 30 patients in the results analysis (10/group).

The study was conducted between September 2018 and April 2019. All patients followed the same treatment protocol and were blinded to which group they belonged.

All cases were performed by a single operator. The treatment procedure followed the same steps for all groups starting by atraumatic extraction, socket debridement, socket grafting, absorbent gelatin sponge coverage, figure 8 propylene $5 / 0$ suture, 6 month postoperative crestal flap, core collection with $3 / 4$ trephine, osteotomy site preparation with implant kit, implant placement, suture, 6 month post-operative prosthetic stage, prosthetic crown delivery.

All patients were periodontally healthy with main reason for extraction being non-restorable tooth due to severe decay.

\section{Inclusion criteria}

- $\quad$ Adult patients with hopeless mandibular molars indicated for extraction and implant placement in two stages that indicate socket preservation.

- $\quad$ Both sexes.

- No intraoral soft and hard tissue pathology.

- $\quad$ No systemic condition that contraindicate implant placement. 


\section{Exclusion criteria}

- $\quad$ Presence of fenestrations or dehiscence of the residual bony Walls after extraction.

- $\quad$ Patients with systemic disease that may affect normal healing.

- $\quad$ Psychiatric problems

- Disorders to implant are related to history of radiation therapy to the head and neck neoplasia, or bone augmentation to implant site.

- $\quad$ Patients that refuse to be called back for implant placement post extraction.

- Patients that have exceeded bone resorption surrounding the tooth prior to extraction and require guided bone regeneration.

- $\quad$ Pregnant Females.

Pre-operative assessment

A thorough preoperative examination of all enrolled candidates was carried out including history taking, clinical and radiographical examination.

\section{Patient interview}

Each patient was interviewed in order to obtain a comprehensive history including a full medical and dental history (Appendix-3).

\section{Clinical examination}

- $\quad$ Patients were inspected for adequate interarch space, periodontal status of the adjacent teeth.

- $\quad$ Soft tissue biotype and nature of the covering mucosa was assessed

- Intraoral photographs and dental impressions were taken. for the selected candidates.

\section{Radiographic examination}

A preoperative digital panoramic radiograph with Panoramic $\mathrm{x}$-ray was taken for each patient as a primary survey to assess and identify the failing teeth, the amount of intra alveolar socket root length of failing teeth, residual alveolar bone between the apex of the socket and alveolar nerve proximity, the direction of the adjacent teeth and the presence of large pre-apical lesion (Figure 1).

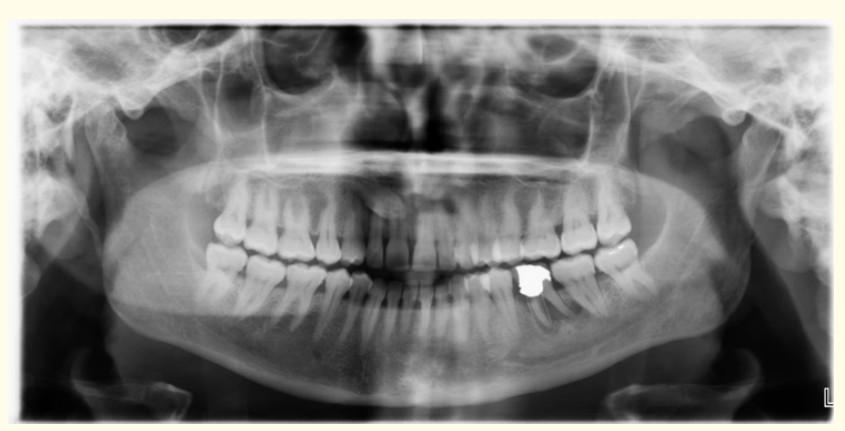

Figure 1: Preoperative Panoramic radiograph (case 3 - ABB group).

\section{Randomization}

The patients were randomly divided using a computer-generated program. Random numbers were used to divide patients into three equal groups of twelve patients according to the material used to fill the alveolar socket post extraction. The test group received Autogenous and Anorganic bovine bone graft mixture while the control-1 group received Anorganic Bovine Bone ${ }^{1}$ alone and the control-2 group received absorbable gelatin sponge alone.

\section{Primary surgical procedure}

Patients were asked to rinse their mouth with chlorohexidine $\mathrm{HCl}$ mouth wash $1.25 \%$ immediately pre-operatively.

All procedures were performed under local anesthesia. All Patients received buccal and lingual infiltration anesthesia using (articane $4 \%$ with adrenaline 1:100000).

Atraumatic extraction of the selected tooth was initiated using sharp periotome to severe the periodontal ligament attachments while preserving the surrounding alveolar bone and interdental papilla and followed by using extraction forceps (Figure 2, 3, 4). 


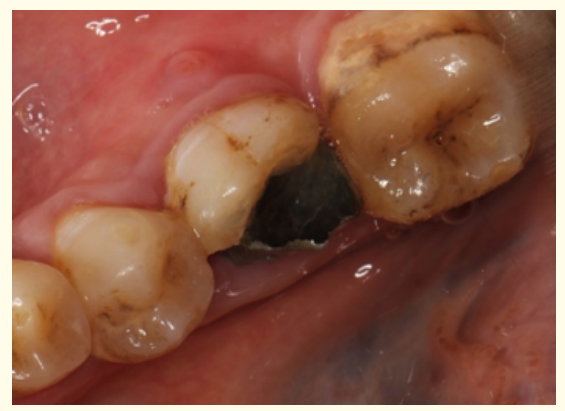

Figure 2: Tooth to be extracted (case 3- ABB group).

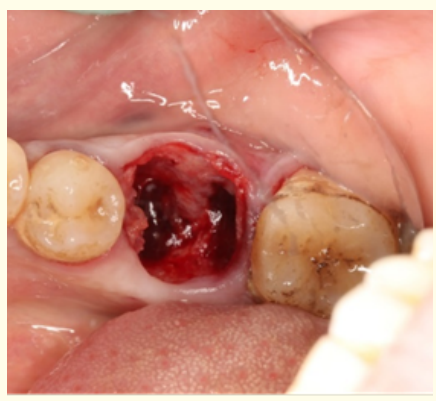

Figure 3: Post extraction socket debridment.

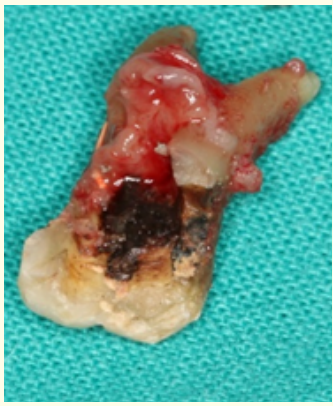

Figure 4: Extracted tooth.

Meticulous mechanical debridement of the extraction socket was carried out using bone currete to remove any soft tissue remnants or granulation tissue. All debris was washed out using copious saline irrigation.
The extraction socket was packed with the grafting material according to the randomization selection of the patient group. The first group was packed with a mixture of approximately equal proportions of anorganic bovine bone and autogenous graft, the second group was filled with the anorganic bovine bone mineral particles alone (Figure 5,6), and the third group was filled with absorbent gelatin sponge. and all the sockets were tightly packed and then covered with a layer of AGS to stabilize the graft particulates inside the socket in cases of the Auto/ABB mixture group or the ABB group (Figure 7,8).

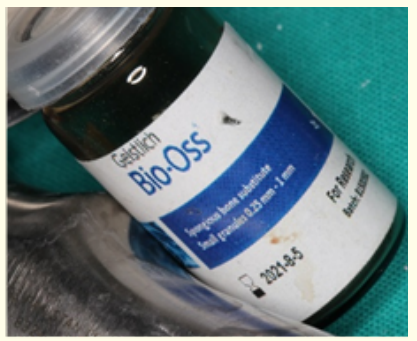

Figure 5: Anorganic Bovine bone minerals vile (o.25-1 mm granules).

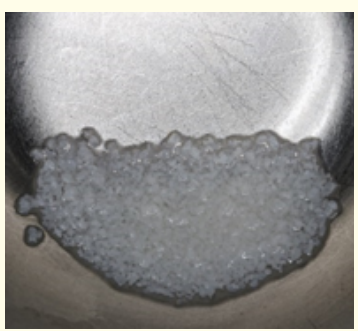

Figure 6: Bovine bone minerals (o.25-1mm granules).

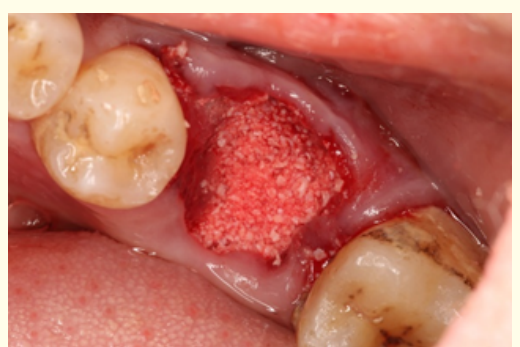

Figure 7: Packing of Xenograft. 


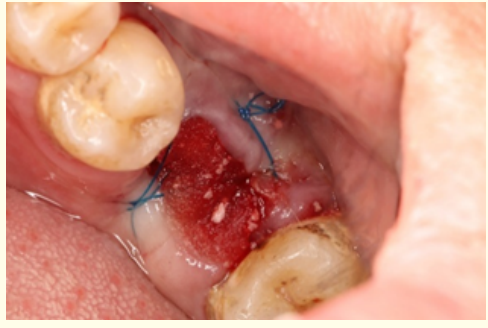

Figure 8: Gelfoam coverage of socket.

Auto/ABB group patients had an extra host surgical site for autogenous bone collection. Submarginal/attached gingival incision $1 \mathrm{~mm}$ above mucogingival junction beveled in the apical direction [50] (Figure 9).

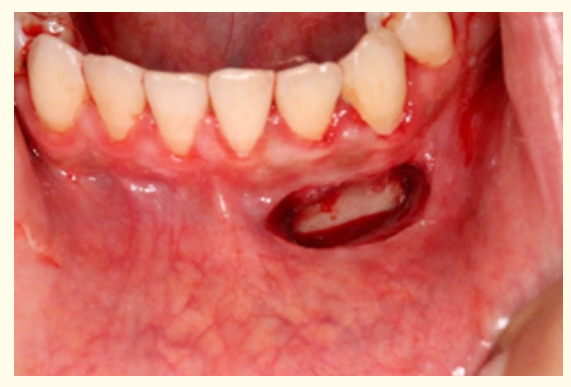

Figure 9: Chin flap for autogenous bone collection using ACM bur.

The harvested graft was collected from the Neo-Biotech ACM Auto Chip Maker [51], The chin flap was sutured with propylene $5 / 0$ suture in continuous suturing manner with lock to prevent dehiscence of flap.

An approximate equal amount of autogenous and Anorganic bovine bone is mixed before packing the mixture in to the grafted alveolar socket.

The sockets were all sutured with propylene $5 / 0$ suture afterwards and the patients were instructed to keep biting on a gauze for one-hour post operatively to ensure bleeding stoppage and clot formation.
Postoperative care and follow up

Postoperatively Patients were instructed to avoid hot dietary for 24 hours, and to have liquid or semiliquid diet for the first 3 days after surgery and to gradually return to their normal diet. The sutures were removed one week postoperative. The healing period was monitored to ensure sustained closure of the grafted site and infection-free regeneration.

After the extraction, patients were prescribed amoxicillin $1 \mathrm{~g}+$ clavulanate potassium ${ }^{2}$, twice per day, for 1 week starting at the day of surgery; metronidazole $500 \mathrm{mg}^{3}$ twice per day for 1 week starting from surgery day; ibuprofen $600 \mathrm{mg}^{4}$, anti-inflammatory/analgesic, twice a day for 2-3 days after surgery; and chlorhexidine $0.12 \%$ mouthwash ${ }^{5}$, oral rinses were prescribed twice a day for 2 weeks.

Post-operative assessment

Clinical assessment

Patients were called for follow-up 1-week post-operative for suture removal and then monthly for six months and were assessed for healing.

\section{Radiographic assessment}

Immediate post-operative cone beam-CT (CBCT-1) was ordered for all patient to act as base line for radiographic analysis.

After 6 months all patients were recalled obtaining a secondary cone beam-CT (CBCT-2) to assess alveolar ridge recession and amount of bone resorption.

\section{Radiographic assessment of alveolar ridge bone loss}

According to researches a close estimate of the bone loss could be measured through a CBCT that is comparable to surgical exploration and can be used for diagnosis of bone defects in periodontal diseases in clinical settings [52].

From both the immediate post-operative (CBCT-1) and 6 months post-operative (CBCT-2) using DICOM viewer software ${ }^{6}$, the measurements were calculated and assessed.

\footnotetext{
${ }^{2}$ Augmenting 1gm, Medical Union Pharmaceuticals (MUP) - Cairo, Egypt, GlaxoSmithKline(gsk) S.A.E. - A.R.E.

${ }^{3}$ Flagyl Sanofi Aventis, Cairo, Egypt.

${ }^{4}$ Brufen, Boots, Cairo, Egypt.

${ }^{5}$ Hexitol, The Arab Drug Company, Cairo, Egypt.

${ }^{6}$ Blue Sky Bio, Blue Sky Bio, LLC, U.S.A
}

Citation: Dina Mohammed Alesawy., et al. "Alveolar Ridge Preservation in Mandibular Molars Using Mixture of Autogenous Bone and Anorganic Bovine Bone (ABB) Versus Anorganic Bovine Bone Alone Versus Absorbent Gelatin Sponge (Randomized Clinical Trial)". Acta Scientific Orthopaedics 4.1 (2021): 56-73. 
The Axial line of the CBCT (blue line) was set on the top of the alveolar ridge of anterior mandibular teeth for CBCT-1 and CBCT-2 (Figure 10).

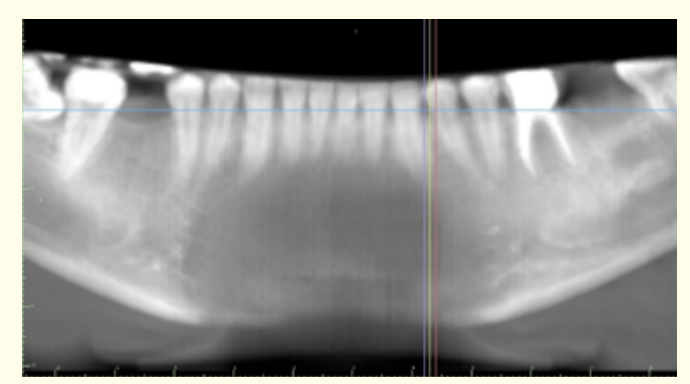

Figure 10: The Axial line of the CBCT (blue line) was set on the top of the alveolar ridge of anterior mandibular teeth.

On this level, the panoramic curve was drawn on the same level in CBCT-1 and CBCT-2 passing through the pulps of anterior mandibular teeth and available posterior teeth (Figure 11).

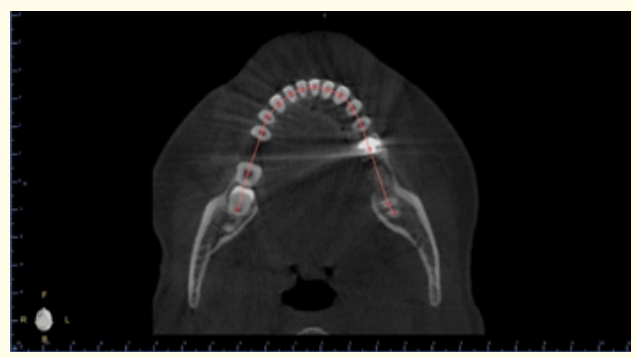

Figure 11: The panoramic curve was drawn on the same level in CBCT-1 and CBCT-2 passing through the pulps of anterior mandibular teeth and available posterior teeth.

The measurements were recorded from the same distance relative to the mental foramen in CBCT- 1 and CBCT- 2 in each case and the differences were calculated to result on the remodeling changes that happened in the alveolar ridge (Figure 12).

For each case, 5 different measurements throughout different positions in the grafted socket was recorded in both cone-beams and the differences were calculated. And the mean difference of the calculated loss was recorded.

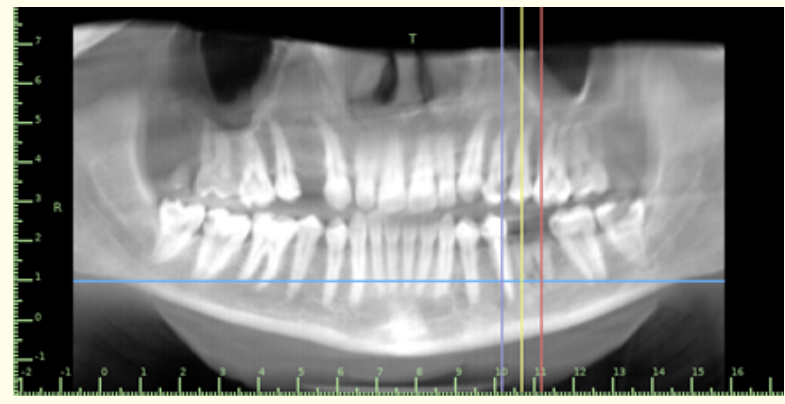

Figure 12: A referenced point of the line coincides with the mental foramen) chosen for identical measurement point between CBCT-1 and CBCT-2 (blue line cross sectional view showing mental foramen while the measured alveolar socket coincides with the yellow line and the distance between the two cross sections is recorded in CBCT-1 and duplicated in CBCT-2 for calculating differences in ridge height and width at the same point).

\section{Measuring technique}

A tangential straight line was made at the highest point of the inferior alveolar canal considering it is a non-changing reference point and two perpendicular lines were made on it from the highest point of the buccal and lingual alveolar plates respectively directly post-extraction and grafting (Figure 13).
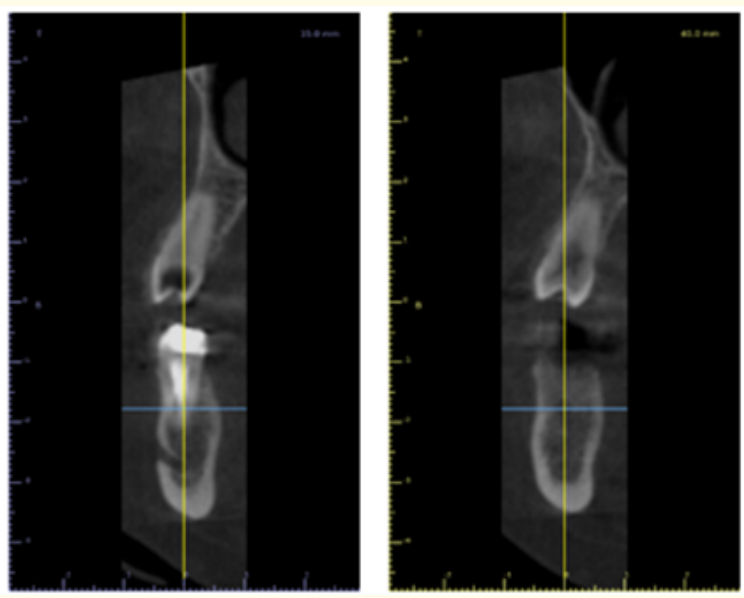

Figure 13: The cross-sectional views with recorded distance in relevance to the mental foramen. CBCT-1. 
A straight line between the two perpendicular lines was made to calculate the bucco-lingual thickness of the top of the ridge ( future implant platform position) (Figure 14).
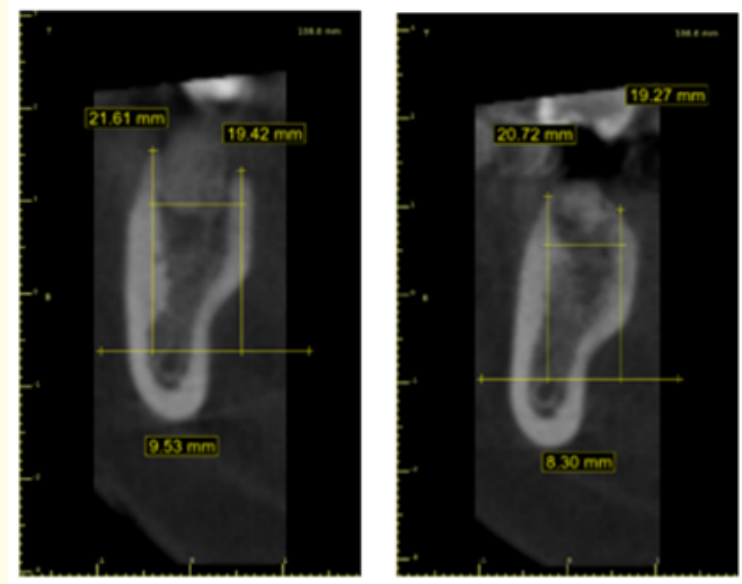

Figure 14: Measurements applied on CBC-1 (a) and CBCT-2 (b) at the same point of the ridge for remodeling calculation.

The same was repeated in the 6- month post-operative CBCT (CBC-2) and the differences were calculated [53].

\section{Second stage surgical procedure}

All patients were recalled 6 months after the grafting procedure to collect core biopsies from the grafted site followed by implant insertion. Crestal flaps were made, followed by trephine bur 3/4 core biopsy claiming (Figure 15). The biopsy sites were then prepared using implant kit drills to the required size and implants were inserted into position then the flap was sutured (Figure 16).

\section{Preparation of the samples and histomorphometric analysis}

After the core biopsies were retrieved, the bony specimens were immediately fixed in $10 \%$ buffered formalin for one week, then decalcified and processed according to the standardized protocol; utilizing Ethylenediaminetetraacetic acid (EDTA)-formic acid combination. Then, the specimens were embedded longitudinally into

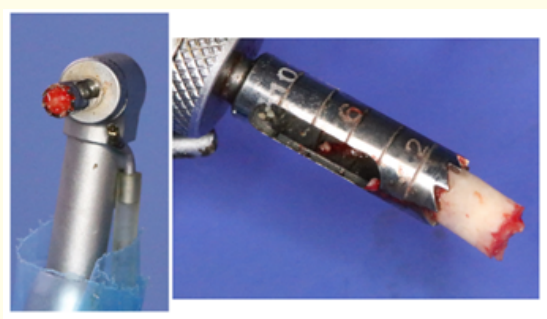

Figure 15: Collected Biopsy from previously grafted site.

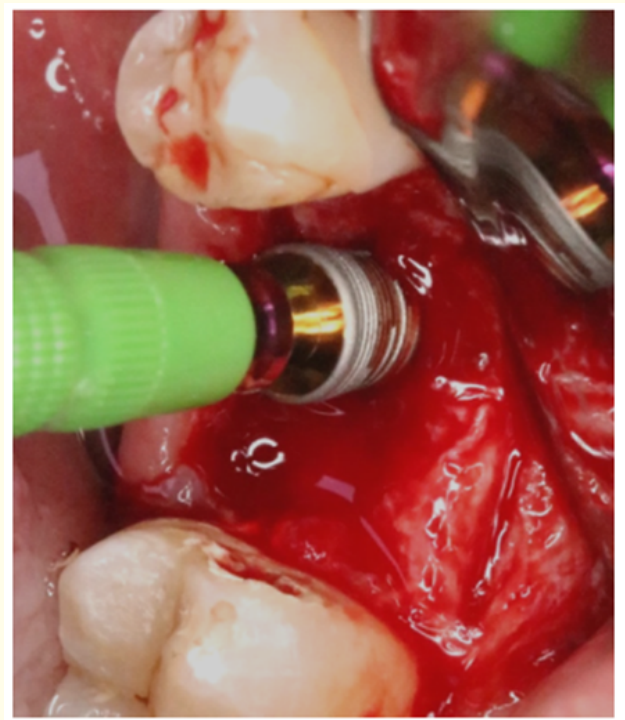

Figure 16: Implant insertion in site of claimed core.

paraffin blocks, which were sliced into longitudinal $5 \mathrm{~mm}$-thick sections using a manual rotary microtome (RM 2135 microtome, Leica, Heidelberger Straße, Nussloch, Germany) and stained with Mayer's hematoxylin and eosin stain (H\&E) for histological analysis.

Histomorphometric Analysis All the stained sections were examined low and high-power light microscopy (Leica, Swizerland). An image analyzer computer system applying the software Leica Quin 500 (Leica Microsystem, Swizerland). For each section, the most representative five fields per specimen were captured using magnification (x100). The bone volume (bone area fraction) was 
measured for each image. Furthermore, the nature and type of the bone present and the intervening marrow was also assessed.

\section{Results}

\section{Demographic data results}

Thirty candidates (17 males, 13 females) suffering from nonrestorable mandibular molar and seeking immediate extraction and delayed implant rehabilitation were selected from the outpatient clinic of Oral and Maxillofacial Surgery Department faculty of Dentistry Cairo University to be included in this study. The present study was approved by the ethics committee the faculty of oral and dental medicine Cairo University.

\section{Clinical results}

A total of 36 patients ( 25 males, 16 females; average age 20 to 60 years) was enrolled in this study, the patients were randomly divided into 3 equal groups using block randomization with stratification (block size: 4) using a formula on Microsoft Excel Software. the mean follow-up was of 6 months from the extraction and socket preservation (1st stage) followed by implant placement, 6-month of follow-up then prosthetic stage and delivery ( $2^{\text {nd }}$ stage) and 1 year follow up for the implants and prosthetics $\left(3^{\text {rd }}\right.$ stage).

6 patients/2 from each group were expelled from the results analysis of the experiment as they were asking to postpone their implant placement surgery for further months that would have affected our 6 month results experiment, making a total of 30 patients (17 males, 13 females) that were included in the study.

All patients signed an informed consent approved by the Cairo University-Faculty of Dentistry. The study was conducted between September 2018 and April 2019. All patients followed the same treatment protocol and were blinded to which group they belonged.

All cases were performed by a single operator. The treatment procedure followed the same steps for all groups starting by atraumatic extraction, socket debridement, socket grafting, absorbent gelatin sponge coverage, figure 8 propylene 5/0 suture, 6 month postoperative crestal flap, core collection with $3 / 4$ trephine, osteot- omy site preparation with implant kit, implant placement, suture, 6 month post-operative prosthetic stage, prosthetic crown delivery.

All patients were periodontally healthy with main reason for extraction being non-restorable tooth due to severe decay. Three patients showed buccal bone fenestration on one of the roots apically followed extraction. No suture dehiscence was recorded in any of the cases following grafting neither following implant placement.

All 36 completely successful cases were recorded, no failures, accidents, adverse events nor poor results were recorded. All extractions healed good and the patients were visiting the clinic every week for four weeks.

All measurements of radiographic CBCT and Histomorphometric analysis were performed by two single blinded examiners. CBCT radiograph performed six months after tooth extraction suggested a good healing of all the sockets.

Radiographic results

\section{Variation of height of buccal alveolar crest}

The lowest variation value of the height of buccal alveolar crest was recorded in the Absorbent gelatin sponge group and the highest variation value was recorded in (Autogenous $+\mathrm{ABB}$ graft) group. One-way analysis of variance (ANOVA) test revealed that the difference between all groups was statistically significant $(\mathrm{P}=0.024)$. Tukey's post hoc revealed no significant difference between (Autogenous + ABB graft) group and ABB graft group, as well as, between $\mathrm{ABB}$ graft and Absorbent gelatin sponge group. Although (Autogenous + ABB graft) group and absorbent gelatin sponge group showed significant difference with each other (Table 1).

Variation of height of lingual/palatal alveolar crest

The lowest variation value of the height of lingual/palatal alveolar crest was recorded in the ABB graft group and the highest variation value was recorded in (Autogenous + ABB graft) group. Oneway analysis of variance (ANOVA) test revealed that the difference between all groups was statistically significant $(\mathrm{P}=0.001)$. Tukey's post hoc revealed no significant difference between (Autogenous + 
Alveolar Ridge Preservation in Mandibular Molars Using Mixture of Autogenous Bone and Anorganic Bovine Bone (ABB) Versus Anorganic Bovine Bone Alone Versus Absorbent Gelatin Sponge (Randomized Clinical Trial)

\begin{tabular}{|c|c|c|c|}
\hline P.o.C & $\begin{array}{c}\text { Autogenous } \\
\text { + ABB graft }\end{array}$ & ABB graft & $\begin{array}{c}\text { Absorbent gelatin } \\
\text { sponge }\end{array}$ \\
\hline Mean & $2.64^{\mathrm{a}}$ & $1.62^{\mathrm{a}, \mathrm{b}}$ & $1.29^{\mathrm{b}}$ \\
\hline Std Dev & 1.48 & 0.87 & 0.71 \\
\hline Std error & 0.49 & 0.29 & 0.24 \\
\hline Max & 5.36 & 3.46 & 2.45 \\
\hline Min & 0.95 & 0.42 & 0.28 \\
\hline F-value & \multicolumn{3}{|c}{4.305} \\
\hline P-value & \multicolumn{3}{|c}{$0.024^{*}$} \\
\hline
\end{tabular}

Table 1: Variation of height of buccal alveolar crest in all groups and significance of the difference using (ANOVA) test.

*significant at $\mathrm{p}<0.05$.

Tukey's post hoc test means sharing the same superscript letter are not significantly different.

ABB graft) and Absorbent gelatin sponge group. While ABB graft group showed significant difference with the other groups (Table 2).

\begin{tabular}{|c|c|c|c|}
\hline P.O.C & $\begin{array}{c}\text { Autogenous + } \\
\text { ABB graft }\end{array}$ & ABB graft & $\begin{array}{c}\text { Absorbent } \\
\text { gelatin sponge }\end{array}$ \\
\hline Mean & $1.59^{\mathrm{a}}$ & $0.48^{\mathrm{b}}$ & $1.44^{\mathrm{a}}$ \\
\hline Std Dev & 0.86 & 0.39 & 0.47 \\
\hline Std error & 0.29 & 0.12 & 0.16 \\
\hline Max & 3.57 & 1.38 & 2.63 \\
\hline Min & 0.57 & 0.01 & 0.85 \\
\hline F-value & \multicolumn{3}{|c}{9.780} \\
\hline P-value & $0.001^{*}$ \\
\hline
\end{tabular}

Table 2: Variation of height of lingual/palatal alveolar crest in all groups and significance of the difference using (ANOVA) test.

*significant at $\mathrm{p}<0.05$.

Tukey's post hoc test means sharing the same superscript letter are not significantly different.

Variation of width of alveolar crest

The lowest variation value of the width of alveolar crest was recorded in the Absorbent gelatin sponge group and the highest

variation value was recorded in (Autogenous + ABB graft) group. One-way analysis of variance (ANOVA) test revealed that the difference between all groups was not statistically significant $(\mathrm{P}=0.063)$. Tukey's post hoc revealed no significant difference between $\mathrm{Au}-$ togenous + ABB graft) group and the other 2 groups. Although (ABB graft) group and absorbent gelatin sponge group showed significant difference with each other (Table 3).

\begin{tabular}{|c|c|c|c|}
\hline P.O.C & $\begin{array}{c}\text { Autogenous } \\
\text { + ABB graft }\end{array}$ & ABB graft & $\begin{array}{c}\text { Absorbent gelatin } \\
\text { sponge }\end{array}$ \\
\hline Mean & $1.63^{\mathrm{a}, \mathrm{b}, \mathrm{c}}$ & $1.55^{\mathrm{a}, \mathrm{b}}$ & $0.89^{\mathrm{a}, \mathrm{c}}$ \\
\hline Std Dev & 0.97 & 0.54 & 0.62 \\
\hline Std error & 0.32 & 0.18 & 0.21 \\
\hline Max & 3.59 & 2.46 & 1.92 \\
\hline Min & 0.22 & 0.55 & 0.08 \\
\hline F-value & \multicolumn{3}{|c}{3.060} \\
\hline P-value & \multicolumn{3}{|c|}{$0.063^{*}$} \\
\hline
\end{tabular}

Table 3: Variation of height of width of alveolar crest in all groups and significance of the difference using (ANOVA) test.

*significant at $\mathrm{p}<0.05$.

Tukey's post hoc test means sharing the same superscript letter are not significantly different.

\section{Radiographic results conclusion}

The variation value of the height of the buccal alveolar crest was lower in the Absorbent gelatin group (1.29 \pm 0.71$)$ compared with the Autogenous $+\mathrm{ABB}(2.64 \pm 1.48)$ and ABB groups $(1.62 \pm 0.87)$ (Figure 17).

The variation value of the height of the lingual/palatal alveolar crest was markedly lower in the ABB group (0.48 \pm 0.39$)$ compared with the Autogenous + ABB group $(1.59 \pm 0.86)$ and Absorbent gelatin group (1.44 \pm 0.47 ) (Figure 17).

The variation value of the width of the alveolar crest was lower in the Absorbent gelatin group $(0.89 \pm 0.62)$ compared with the Autogenous + ABB (1.63 \pm 0.97$)$ and ABB groups (1.55 \pm 0.54$)$ (Figure 17). 
Alveolar Ridge Preservation in Mandibular Molars Using Mixture of Autogenous Bone and Anorganic Bovine Bone (ABB) Versus Anorganic Bovine Bone Alone Versus Absorbent Gelatin Sponge (Randomized Clinical Trial)

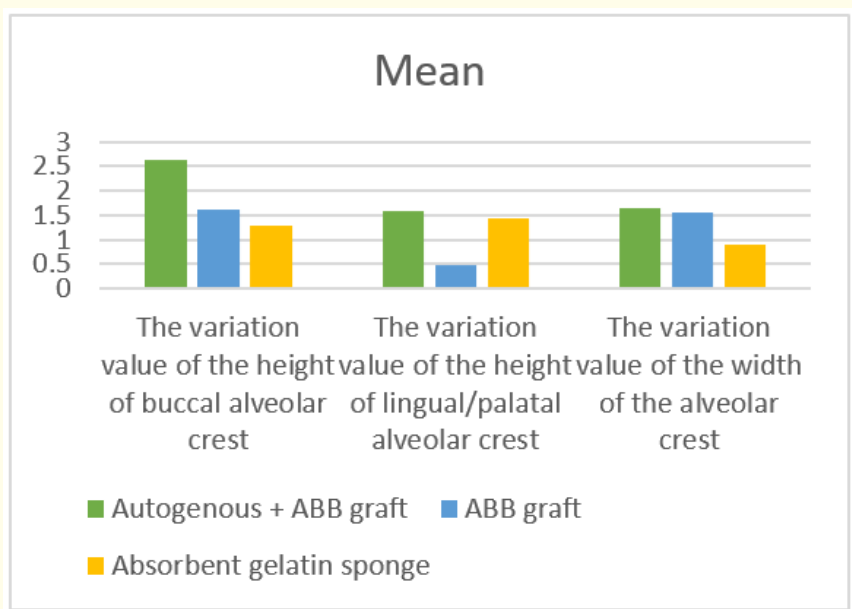

Figure 17: Column chart showing variation values of the buccal and lingual/palatal alveolar crest height and the alveolar crest width in the all groups.

\section{Histomorphometric results}

\section{Bone area percent}

The greatest mean area percent was recorded in Absorbent gelatin sponge group, whereas the lowest value was recorded in (Autogenous + ABB graft) group. One-way analysis of variance (ANOVA) test revealed that the difference between all groups was statistically significant $(\mathrm{P}<0.001)$. Tukey's post hoc revealed no significant difference between (Autogenous + ABB graft) group and $\mathrm{ABB}$ graft group. While absorbent gelatin sponge group showed significant difference with the other groups (Table 4).

\begin{tabular}{|c|c|c|c|}
\hline P.O.C & $\begin{array}{c}\text { Autogenous + } \\
\text { ABB graft }\end{array}$ & ABB graft & $\begin{array}{c}\text { Absorbent } \\
\text { gelatin sponge }\end{array}$ \\
\hline Mean & $42.34^{\mathrm{a}}$ & $48.42^{\mathrm{a}}$ & $58.88^{\mathrm{b}}$ \\
\hline Std Dev & 8.67 & 4.88 & 8.13 \\
\hline Std error & 2.89 & 2.26 & 2.71 \\
\hline Max & 57.44 & 55.94 & 67.64 \\
\hline Min & 30.58 & 38.23 & 43.22 \\
\hline F-value & \multicolumn{3}{|c}{12.720} \\
\hline P-value & \multicolumn{3}{|c}{$0.001^{*}$} \\
\hline
\end{tabular}

Table 4: Area percent of bone in all groups and significance of the difference using (ANOVA) test.

*significant at $\mathrm{p}<0.05$.

Tukey's post hoc test means sharing the same superscript letter are not significantly different.
Area percent of residual material

The greatest mean area percent was recorded in (Autogenous + ABB graft) group, whereas the lowest value was recorded in Absorbent gelatin sponge group. One-way analysis of variance (ANOVA) test revealed that the difference between all groups was statistically significant $(\mathrm{P}<0.001)$. Tukey's post hoc revealed no significant difference between $\mathrm{ABB}$ graft group and Absorbent gelatin sponge group. While (Autogenous + ABB graft) group showed significant difference with the other groups (Table 5).

\begin{tabular}{|c|c|c|c|}
\hline P.O.C & $\begin{array}{c}\text { Autogenous }+ \\
\text { ABB graft }\end{array}$ & ABB graft & $\begin{array}{c}\text { Absorbent } \\
\text { gelatin sponge }\end{array}$ \\
\hline Mean & $32.19^{\mathrm{a}}$ & $20.87^{\mathrm{b}}$ & $20.36^{\mathrm{b}}$ \\
\hline Std Dev & 3.22 & 3.09 & 4.77 \\
\hline Std error & 1.07 & 1.03 & 1.59 \\
\hline Max & 37.87 & 15.67 & 13.54 \\
\hline Min & 27.22 & 25.65 & 27.34 \\
\hline F-value & 31.445 \\
\hline P-value & \multicolumn{3}{|c}{$0.001^{*}$} \\
\hline
\end{tabular}

Table 5: Area percent of residual material in all groups and significance of the difference using (ANOVA) test

*significant at $\mathrm{p}<0.05$.

Tukey's post hoc test means sharing the same superscript letter are not significantly different.

\section{Discussion}

The primary aim of this study was to assess the reliability of different types of grafting materials in socket preservation to minimalize the bone resorption which takes place during the socket remodeling phase. The secondary aim was to evaluate which of the grafting materials used would produce a better bone quality histologically and the resorption durability of this graft in the time phase of the study which is 6 months.

In the current study the patients all had a standard similar operation procedure of extraction of hopeless mandibular molar teeth, socket curettage, grafting, socket coverage with an AGS layer to preserve the grafting material, suturing and receiving a cone beam x-ray as a primary record for the socket. After six month a secondary CBCT was made, followed by a secondary surgical proce- 
dure in the form of a crestal flap followed by core biopsy collection and implant installation. All the patients had the same after care measurements and precautions. The results were assessed radiographically and histomorphometrically.

The decrease of alveolar crest width in ABB group in our study was $(1.55 \pm 0.54 \mathrm{~mm})$, The loss of bone in the buccal bone plate was $(1.62 \pm 0.87 \mathrm{~mm})$, the lingual plate $(0.48 \pm 0.39 \mathrm{~mm})$. The mean area percent of available bone was (48.42\%). The mean area percent of residual material $(20.87 \%)$.

The difference in the vertical reduction between what he found and ours could be due to the fact that we measured the buccal and lingual bone plates separately. The buccal bone reduction is highly dependent on the width of the buccal bone plate [54].

The bundle bone surrounded the tooth is thought to have an inevitable resorption as it's a tooth dependent structure [55] If the buccal bone plate is thin enough to be formed of mainly bundle pone, the loss of periodontal ligament blood supply would mean the resorption of buccal bone plate [56].

The difference between the loss in the buccal and lingual bone plate has been attributed to the phenomena that the buccal bone is usually lesser in width than that of the buccal bone [57].

histomorphometric differences could be due to their use of porcine graft that had a higher residual graft material results supporting our suggestion that porcine could have slower rate of resorption than the $\mathrm{ABB}$ we used, or it might be a matter of difference in the molecular structure of both xenogeneic materials. The same goes for the difference in available bone measurements, since the micro-molecular structure of the ABB we used allows for more space of new bone development.

In relation to Autogenous and $\mathrm{ABB}$ mixture, our study showed a loss of bone in the buccal bone plate was the highest of $2.64 \pm 1.48$, The loss of bone in the lingual plate of $1.59 \pm 0.86$, The decrease in alveolar crest width also recorded to the highest equal to 1.63 \pm 0.97 .

The autogenous bone was thought to retard the action of the $\mathrm{ABB}$ since its resorption rate is known to be equal to that of the socket wall bone if not higher as a result of dead cells received in the autogenous graft.

Results of our study in relation to the Autogenous addition to the ABB was not promising since it had a higher resorption than the ABB alone. While their resorption from the site might have affected the degree of degradation of the ABB which kept the osteoclastic activity on the Autogenous bone resulting on higher residual material results on the Auto/ABB group.

The Auto/ABB group had the least bone area percent and the highest residual material percent. that would be applicable for the fact that the autogenous bone retarded the effect of $A B B$ as the results of the ABB alone were better in comparison.

The greatest mean area percent was recorded in Absorbent gelatin sponge group, while the histological analysis revealed no significant difference between Autogenous/ABB graft group and ABB graft group.

The mean area percent of residual material lowest value in the present study was recorded in Absorbent gelatin sponge group with also favorable. It implies that the use of AGS socket graft material could be promising. The results could be due to the fact that the other two groups had ABB which is known to have a very low resorption and biodegradation rate. While the Gelatin is a highly bioresorbable material [39].

\section{Summary}

The present study evaluated the effect of Autogenous and Anorganic Bovine Bone (Auto/ABB) mixtures versus the Anorganic Bovine Bone Mixture versus the Absorbent Gelatin Sponge on the effect of Alveolar socket preservation.

The study was conducted on 30 patients (10 in each group). The patients ages were from second decade to the fifth decade. All patients passed through the same surgical procedure of atraumatic extraction and grafting. All patients were recalled second day after the operation then weekly for the first month followed by monthly recall for 6 months postoperative till implant placement.

The clinical evaluation included assessment of postoperative sequalae including edema, pan, and postoperative healing. Radio-

Citation: Dina Mohammed Alesawy., et al. "Alveolar Ridge Preservation in Mandibular Molars Using Mixture of Autogenous Bone and Anorganic Bovine Bone (ABB) Versus Anorganic Bovine Bone Alone Versus Absorbent Gelatin Sponge (Randomized Clinical Trial)". Acta Scientific Orthopaedics 4.1 (2021): 56-73. 
graphic evaluation involved CBCT radiographs taken for every patient immediately post extraction and grafting and 6 month postoperatively.

The measuring criteria were Cone Beam CT radiographical buccal, lingual and horizontal width dimension of the alveolar socket directly after extraction and 6 months after grafting. The second measurements carried where the histomorphometrical mean bone percentage and available residual bone after 6 months of grafting.

The Auto/ABB loss of bone in the buccal bone plate was the highest of $2.64 \pm 1.48 \mathrm{~mm}$, The loss of bone in the lingual plate of $1.59 \pm 0.86 \mathrm{~mm}$, The decrease in alveolar crest width also recorded to the highest equal to $1.63 \pm 0.97 \mathrm{~mm}$. The histomorphometric mean bone area percent was least of $42.34 \%$ and the highest residual material percent of $32.19 \%$. The results of this group were considered unpromising, and the addition of ABB to the autogenous bone was considered to have a retarding effect to the solo use of each graft. Other materials need to be considered in relation to socket grafting giving in account the donor sites morbidity and patients suffering in the process of acquiring the autogenous bone.

The ABB grafted sited results where a mean buccal loss of $1.62 \mathrm{~mm}$, lingual loss of $0.48 \mathrm{~mm}$ and horizontal loss $1.55 \mathrm{~mm}$. The histological mean bone area percent was $48.42 \%$ and residual percent of $20.87 \%$. The results were considered successful and the ABB fulfilled the aim of preventing the socket collapse in the 6 months. Considerations should be noted since the residual material of the $A B B$ is expected to last for a long time due to its low resorbability that might have a future effect on installed implant in relation to Bone-Implant contact and osseointegration.

The AGS grafted sites showed promising results with a mean buccal loss of $1.29 \mathrm{~mm}$, lingual $1.44 \mathrm{~mm}$ and horizontal $0.89 \mathrm{~mm}$. The Bone area percent was the highest equal to $58.88 \%$ and a residual of $20.36 \%$ as the least of the examined groups. The results were thought to be due to the fact that the AGS promoted the natural healing of the socket while aiding in stabilizing the blood clot and maintaining the blood cells required for rapid healing and osteoblastic activity while preventing the soft tissue creeping into the socket. The material needs more researches to prove it valuable for socket preservation, giving into account its ease of usage to all operators and cost effectiveness for patients.

\section{Conclusion}

Autogenous and anorganic bovine bone mixture showed inferior result regarding the amount of new bone formation, the amount of residual graft, and the amount of radiographic bone loss in comparison to the anorganic bovine bone alone and absorbent gelatin sponge in the socket grafting procedure. Giving consideration to the hassle of autogenous bone acquisition to the operator and patient.

Anorganic Bovine bone results showed good radiographical and histological results in consideration of using it as a ridge preservation material.

Absorbent Gelatin Sponge showed superior results for the ridge preservation with a high amount of bone formation, low residual remnants and acceptable radiographic bone loss in relation to the other two materials used. Giving into account its ease of application and cost effectiveness.

Awareness should be afforded to all operators and passing to patients on the importance of socket preservation and atraumatic extractions.

Further researches are required to validate our results on all three groups.

\section{Bibliography}

1. B Shakibaie. "Socket and ridge preservation from the threedimensional perspective". (2009): 24-33.

2. P R Schmidlin., et al. "Prevention of alveolar ridge resorption after tooth extraction--a review". Schweizer Monatsschrift fur Zahnmedizin $=$ Rev. Mens. suisse d'odonto-stomatologie $=$ Riv. Mens. Svizz. di Odontol. e Stomatol 114.4 (2004): 328-336.

3. MG Araújo., et al. "Alveolar socket healing: what can we learn?". Periodontology 1 (2015): 122-134.

4. GA Kumar. "Criteria for immediate placement of oral implantsa mini review Biology and Medicine Criteria for immediate placement of oral implants-a mini review" (2012).

5. Schropp L., et al. "Bone healing and soft tissue contour changes following single-tooth extraction: a clinical and radiographic 12-month prospective study". International Journal of Periodontics and Restorative Dentistry 23.4 (2003): 313-323. 
6. P G Carlsson GE. "Morphologic changes of the mandible after extraction and wearing of dentures. A longitudinal, clinical, and x-ray cephalometric study covering 5 ye". Odontology Review 18.1 (1967): 27-54.

7. P Mercier and R Lafontant. "Residual alveolar ridge atrophy: Classification and influence of facial morphology". Journal of Prosthetic Dentistry 41.1 (1979): 90-100.

8. D A Atwood. "Some clinical factors related to rate of resorption of residual ridges". Journal of Prosthetic Dentistry 12.3 (1962): 441-450.

9. Q Gu., et al. "Macrophages and bone inflammation". Journal of Orthopaedic Translation 10 (2017): 86-93.

10. C Baat., et al. "Factors connected with alveolar bone resorption among institutionalized elderly people". Community Dentistry and Oral Epidemiology 21.5 (1993): 317-320.

11. F S., et al. "Histological comparison of healing extraction sockets implanted with bioactive glass or demineralized freezedried bone allograft: A pilot study". Journal of Periodontology 73.1 (2002).

12. Gabriel Zubillaga., et al. "Changes in alveolar bone height and width following post-extraction ridge augmentation using a fixed bioabsorbable membrane and demineralized freezedried bone osteoinductive graft". Journal of Periodontology 74.7 (2003): 965-975.

13. A Z and N CE. "The application of deproteinized bovine bone mineral for ridge preservation prior to implantation. Clinical and histological observations in a case report". Journal of Periodontology 69.9 (1998): 1062-1067.

14. C D., et al. "Healing of human extraction sockets filled with BioOss". Clinical Oral Implants Research 14.2 (2003): 137-143.

15. S GKB., et al. "Preservation of ridge dimensions following grafting with coral granules of 48 post-traumatic and postextraction dento-alveolar defects". Dental Traumatology 19.4 (2003): 221-227.
16. G R., et al. "Medial-grade calcium sulfate hemihydrate (surgiplaster) in healing of a human extraction socket--histologic observation at 3 months: a case report". The International Journal of Oral and Maxillofacial Implants 20.4 (2005): 636-641.

17. V Lekovic., et al. "Preservation of Alveolar Bone in Extraction Sockets Using Bioabsorbable Membranes". Journal of Periodontology 69.9 (1998): 1044-1049.

18. F Diès., et al. "Bone regeneration in extraction sites after immediate placement of an e-PTFE membrane with or without a biomaterial: A report on 12 consecutive cases". Clinical Oral Implants Research (1996).

19. Hoffmann., et al. "Alveolar Bone Preservation in Extraction Sockets Using Non-Resorbable dPTFE Membranes: A Retrospective Non-Randomized Study". Journal of Periodontology 79.8 (2008): 1355-1369.

20. I JM., et al. "Ridge preservation with freeze-dried bone allograft and a collagen membrane compared to extraction alone for implant site development: a clinical and histologic study in humans". Journal of Periodontology 74.7 (2003): 990-999.

21. D Cardaropoli and G Cardaropoli. "Preservation of the postextraction alveolar ridge: a clinical and histologic study". International Journal of Periodontics and Restorative Dentistry (2008).

22. D Laurito., et al. "Alveolar Ridge Preservation with nc-HA and d-PTFE Membrane: A Clinical, Histologic, and Histomorphometric Study". International Journal of Periodontics and Restorative Dentistry 37.2 (2017): 283-290.

23. C Laurencin., et al. "Bone graft substitutes". Expert Review of Medical Devices 3.1 (2006): 49-57.

24. B Kyle. "Implant Site Development and Extraction Site Grafting" (2011).

25. T Albrektsson and C Johansson. "Osteoinduction, osteoconduction and osseointegration". European Spine Journal 10 (2001): S96-S101.

26. P V Giannoudis., et al. "Bone substitutes: An update". Injury 36.3 (2005): S20-S27. 
Alveolar Ridge Preservation in Mandibular Molars Using Mixture of Autogenous Bone and Anorganic Bovine Bone (ABB) Versus Anorganic Bovine Bone Alone Versus Absorbent Gelatin Sponge (Randomized Clinical Trial)

27. C S and MG Zeeshan Sheikh. "Bone Replacement Materials and Techniques Used for Achieving Vertical Alveolar Bone Augmentation". Materials (Basel) 8 (2015): 2953-2993.

28. R E Marx. "Clinical application of bone biology to mandibular and maxillary reconstruction". Clinics in Plastic Surgery 21.3 (1994): 377-392.

29. G J Zipfel., et al. "Bone grafting". Neurosurgery Focus 14.2 (2008): e8.

30. J A Obwegeser. "Absorbable and bioconvertible osteosynthesis materials in maxillofacial surgery". Mund. Kiefer. Gesichtschir 2.6 (1998): 288-308.

31. H Burchardt. "The biology of bone graft repair". Clinical Orthopaedics and Related Research 174 (1983): 28-42.

32. MR Urist. "Bone: Formation by Autoinduction". Science 150.3698 (1965): 893-899.

33. A Barone., et al. "Clinical and Histological changes after ridge preservation with two xenografts: preliminary results from a multicentre randomized controlled clinical trial". Journal of Clinical Periodontology 44.2 (2017): 204-214.

34. R U Light and H R Prentice. "Surgical Investigation of a New Absorbable Sponge Derived from Gelatin for Use in Hemostasis". Journal of Neurosurgery 2.5 (1945): 435-455.

35. A Smith. "NEW AND NONOFFICIAL REMEDIES". Council on Pharmacy and Chemistry 135.14 (1947): 921.

36. M Hoque., et al. "Gelatin Based Scaffolds for Tissue Engineering-a Review". Journal of Polymer Research 9.1 (2015): 15.

37. D Olsen., et al. "Recombinant collagen and gelatin for drug delivery". Advanced Drug Delivery Reviews 55.12 (2003): 15471567.

38. R A de la Torre., et al. "Hemostasis and hemostatic agents in minimally invasive surgery". Surgery 142.4 (2007): S39-S45.

39. K B Djagny., et al. "Gelatin: A Valuable Protein for Food and Pharmaceutical Industries: Review". Critical Reviews in Food Science and Nutrition 41.6 (2001): 481-492.
40. M Meyer. "Processing of collagen based biomaterials and the resulting materials properties". BioMedical Engineering OnLine 18.1 (2019)24.

41. S Gorgieva and V Kokol. "Collagen- vs. Gelatine-Based Biomaterials and Their Biocompatibility: Review and Perspectives". in Biomaterials Applications for Nanomedicine, InTech (2011).

42. W C Guralnick and L Berg. "Gelfoam in oral surgery". Oral Surgery, Oral Medicine, Oral Pathology, and Oral Radiology 1.7 (1948): 632-639.

43. N A Faculty. "Efficacy of Chitosan and Absorbable Gelatin Sponge on Hemostasis and Wound Healing Following Tooth Extraction 'a Comparative Study”. (2016).

44. Elisabettacenni., et al. "Biocompatibility and performance in vitro of a hemostatic gelatin sponge". Journal of Biomaterials Science, Polymer Edition 11.7 (2000): 685-699.

45. D Timothy Kosinski. "A Simple and Cost-Effective Socket Preservation Technique". 35.4 (2016): 90.

46. M C Echave., et al. "Gelatin as Biomaterial for Tissue Engineering”. Current Pharmaceutical Design 23.24 (2017): 3567-3584.

47. R Rohanizadeh., et al. "Gelatin sponges (Gelfoam $®$ ) as a scaffold for osteoblasts". Journal of Materials Science: Materials in Medicine 19.3 (2008): 1173-1182.

48. L Bodner. "Effect of decalcified freeze-dried bone allograft on the healing of jaw defects after cyst enucleation". Journal of Oral and Maxillofacial Surgery 54.11 (1986): 1282-1286.

49. MD Finn., et al. "Osseous regeneration in the presence of four common hemostatic agents". Journal of Oral and Maxillofacial Surgery 50.6 (1992): 608-612.

50. A Desai., et al. "Current concepts and guidelines in chin graft harvesting: A literature review". International Journal of Oral Health Sciences 3.1 (2013): 16.

51. "Neo-Biotech ACM Auto Chip Maker - Swallow Dental".

52. D Goodarzi Pour., et al. "Accuracy of Cone Beam Computed Tomography for Detection of Bone Loss". Journal of Dentistry (Tehran) 12.7 (2015): 513-523. 
53. P Wenjian Zhang., et al. "Cone Beam Computerized Tomography Measurement of Alveolar Ridge at Posterior Mandible for Implant Graft Estimation". Journal of Oral Implantology XLI.6 (2015): 231-237.

54. D Cardaropoli. "Ridge Preservation-Dealing with extraction sockets" (2018).

55. V Chappuis., et al. "Clinical relevance of dimensional bone and soft tissue alterations post-extraction in esthetic sites". Periodontology 73.1 (2000): 73-83.

56. "Bone healing and soft tissue contour changes following single-tooth extraction: A clinical and radiographic 12-month prospective study". Journal of Prosthetic Dentistry 91.1 (2004): 92.

57. M G Araújo and J Lindhe. "Dimensional ridge alterations following tooth extraction. An experimental study in the dog". Journal of Clinical Periodontology 32.2 (2005): 212-218.

\section{Assets from publication with us}

- Prompt Acknowledgement after receiving the article

- Thorough Double blinded peer review

- Rapid Publication

- Issue of Publication Certificate

- High visibility of your Published work

Website: https://www.actascientific.com/

Submit Article: https://www.actascientific.com/submission.php

Email us: editor@actascientific.com

Contact us: +919182824667 